\title{
PULSEIRA HEMOSTÁTICA TR BAND®: VANTAGENS DO USO PÓS CATETERISMO RADIAL E ASSISTÊNCIA DE ENFERMAGEM
}

\section{ARTIGO DE REVISÃO}

D'AMICO, Mariana Thayla de Souza ${ }^{1}$

LIMA, Itamara Farias de ${ }^{2}$

SILVA, lana Virgínia Monteiro $\mathrm{Da}^{3}$

ARAÚJO, Joyce Fernandes ${ }^{4}$

MORAIS, Alceni ${ }^{5}$

D’AMICO, Mariana Thayla de Souza. Et al. Pulseira hemostática $\operatorname{Tr}$ Band®: Vantagens do uso pós cateterismo radial e assistência de enfermagem. Revista Científica Multidisciplinar Núcleo do Conhecimento. Ano 04, Ed. 10, Vol. 05, pp. 164173. Outubro de 2019. ISSN: 2448-0959, Link de acesso: https://www.nucleodoconhecimento.com.br/saude/pulseira-hemostatica

\section{RESUMO}

Introdução: O cateterismo cardíaco é um procedimento invasivo, realizado para fins diagnósticos, intervencionistas e tratamento nas artérias coronárias. A utilização da artéria radial para realização deste procedimento tem sido cada vez mais estudada

\footnotetext{
1 Discente da graduação de Enfermagem da Universidade Anhembi Morumbi (UAM).

2 Discente da graduação de Enfermagem da Universidade Anhembi Morumbi (UAM).

${ }^{3}$ Discente da graduação de Enfermagem da Universidade Anhembi Morumbi (UAM).

${ }^{4}$ Discente da graduação de Enfermagem da Universidade Anhembi Morumbi (UAM).

${ }^{5}$ Mestre em ciências da saúde pela Universidade Federal de São Paulo (UNIFESP). Docente do curso de graduação pela Universidade Anhembi Morumbi (UAM) e pósgraduação pela Faculdade Israelita de Ciências da Saúde Albert Einstein.
} 
como primeira opção de utilização. Seu custo comparado à outras vias, concomitantemente aos curativos utilizados demonstram valores mais acessíveis com a utilização da arterial radial e da pulseira hemostática TR Band®. Objetivo: Identificar na literatura os benefícios da utilização da pulseira hemostática na via radia e a assistência de enfermagem. Método: Trata-se de um estudo realizado através de revisão bibliográfica. Foram incluídos artigos publicados no período compreendido entre 2010 e 2019 nos idiomas português, russo e inglês. A busca foi realizada nas seguintes bases de dados: Literatura Latino-Americana e do Caribe em Ciências da Saúde (LILACS) e Banca Virtual de Saúde (BVS) e nos seguintes periódicos eletrônicos: Scientific Electronic Library On Line (SCIELO). Salienta-se que as palavras-chave utilizadas foram baseadas nos descritores: Hemostasia/ Hemostasis/ Hemostasis; Terapêutica/ Terapéutica/ Therapeutics; Cateterismo/ Cateterismo/ Catheterization; Artéria radial/ Arteria radial/ Radial Artery; Conforto do paciente/ Comodidad del Paciente/ Patient Comfort. Resultados: Foram analisados cinco artigos relacionados à temática, das bases de dados LILCAS, BVS e SciElo, entre os anos de 2010 e 2019. Constatou-se que a utilização da pulseira compressiva TR Band® vem sendo cada vez mais utilizada para o curativo de cateterismo cardíacos pela via radial. Conclusão: É uma forma de curativo eficaz e cada vez mais implantadas por protocolos com a finalidade de manter a hemostasia após o procedimento realizado na artéria radial.

Palavras-chave: Hemostasia, terapêutica, cateterismo, artéria radial, conforto do paciente.

\section{INTRODUÇÃO}

O cateterismo cardíaco é um procedimento invasivo que combina a avaliação hemodinâmica e angiográfica de diferentes estruturas cardíacas com vista ao diagnóstico e/ou a intervenção de patologias do sistema cardiovascular. ${ }^{1}$

A utilização da artéria radial para procedimento coronário invasivo (ICP) é de grande interesse por delimitar inúmeras vantagens, entre elas estão o conforto do paciente após o procedimento, retorno mais rápido em suas atividades, diminuição do período 
de internação e gastos hospitalares, e diminuição nos índices de intercorrências no local da punção em comparação com a artéria femoral, reduzindo o risco de hemorragia, morte e morbimortalidade. ${ }^{2}$

Diversos estudos na literatura atual apresentam diversas formas de hemostasias realizada através de diferentes tipos de curativos. O curativo compressivo realizado apenas de gaze e micropore é ainda um dos meios mais utilizados para realização da hemostasia pós ICP, entretanto, constatamos seu índice em termos, elevados, referente à obstrução de artéria radial (OAR) em relação à pulseira hemostática TR Band $\AA^{3}{ }^{3}$

Por sua vez, a utilização da pulseira hemostática TR Band® vêm sendo adotada pelas instituições por meio de protocolos que visam garantir um melhor resultado hemostático, maior conforto ao paciente, melhor maneira de manuseio e melhor visibilidade de possíveis sinais flogísticos em comparação do curativo compressivo simples. ${ }^{4}$

Alguns autores contrapõem estas afirmações em sentenças negativas referente à utilização da pulseira em comparação ao curativo simples, destacando o preço da pulseira bem como, maiores índices de hematomas de grau I e II, OAR, espasmos, hemorragias leves e pseudo-aneurismas. ${ }^{5}$

As patologias mais observadas são aquelas que necessitam intervenções coronarianas por vias arteriais, dentre elas, o procedimento coronário de maior incidência é o cateterismo cardíaco. O objetivo deste estudo foi determinar os benefícios da utilização da pulseira hemostática TR Band®, visando a obtenção da hemostasia em pacientes submetidos a ICP em acesso transradial (TRA). Durante o levantamento dos estudos, foram encontrados prós e contras em relação a utilização da pulseira hemostática, e destacando os aspectos em relação ao uso da pulseira hemostática, seus benefícios e a assistência de enfermagem ao final da ICP. Após analisar a fundo, tendo em vista a comparação com outros curativos usuais, a utilização da TR Band® sobressai em grandes pontos como prevenção de OAR, melhor manuseio e menos demanda ao profissional de enfermagem. ${ }^{6}$ 


\section{OBJETIVO}

Identificar na literatura os benefícios da utilização da TR Band® na via radial e assistência de enfermagem.

\section{MÉTODO}

\subsection{TIPO DE ESTUDO}

Tratou-se de um estudo realizado por meio de revisão bibliográfica, método que diz respeito ao levantamento da literatura de relevância, já publicada em revista, publicação avulsa e imprensa escrita sobre o tema em questão, que serve para embasar a investigação referente ao estudo proposto. Foi realizado um levantamento bibliográfico de artigos sobre vantagens do uso da pulseira hemostática TR Band® pós cateterismo radial e assistência de enfermagem publicados nos idiomas português, espanhol e inglês. Foram incluídos artigos publicados no período compreendido entre 2010 e 2019 nos idiomas português e inglês. A busca foi realizada nas seguintes bases de dados: Literatura Latino-Americana e do Caribe em Ciências da Saúde (LILACS), Banca Virtual de Saúde (BVS), e National Library of Medicine, USA (MEDLINE) e nos seguintes periódicos eletrônicos: Scientific Electronic Library On Line (SCIELO).

Salienta-se que as palavras-chave utilizadas foram baseadas nos Descritores em Ciências da Saúde (DECS).

Relacionados à temática proposta, foram levantados 8 artigos que discutem sobre a utilização da pulseira hemostática TR BAND® e a assistência de enfermagem. A relação dos achados bibliográficos para este estudo se encontra no Quadro 1.

Quadro 1. Bibliografias potencias acerca do tema: "Pulseira hemostática TR BAND®: vantagens do uso pós cateterismo radial e assistência de enfermagem".

\section{\begin{tabular}{l|l|l|l} 
Autores Ano Título & Base de dados/Revista
\end{tabular}}




\begin{tabular}{|c|c|c|c|}
\hline $\begin{array}{l}\text { Erlley } \\
\text { Raquel } \\
\text { Aragão } \\
\text { Nobrega } \\
1\end{array}$ & $\begin{array}{l}201 \\
6\end{array}$ & $\begin{array}{l}\text { Hemostasia da } \\
\text { artéria radial } \\
\text { pós cateterismo } \\
\text { cardíaco: } \\
\text { comparação } \\
\text { randomizada } \\
\text { do tempo de } \\
\text { compressão e } \\
\text { avaliação das } \\
\text { complicações } \\
\text { vasculares }\end{array}$ & $\begin{array}{l}\text { https://repositorio.ufpe.br/handle/123456789/1 } \\
8942\end{array}$ \\
\hline $\begin{array}{l}\text { Dall'Ort } \\
\text { o CC, et } \\
\text { al. }^{2}\end{array}$ & $\begin{array}{l}201 \\
0\end{array}$ & $\begin{array}{l}\text { Angioplastia } \\
\text { Coronária nas } \\
\text { Indicações Off- } \\
\text { Label: } \\
\text { Comparação } \\
\text { das Vias Radial } \\
\text { vs. Femoral }\end{array}$ & LILACS/BVS \\
\hline $\begin{array}{l}\text { S. Assif } \\
\text { Neto et } \\
\text { al. }^{3}\end{array}$ & $\begin{array}{l}201 \\
5\end{array}$ & $\begin{array}{l}\text { Comparação } \\
\text { do curativo } \\
\text { compressivo } \\
\text { vs. Pulseira } \\
\text { hemostática } \\
\text { após } \\
\text { cateterização } \\
\text { por via radial. }\end{array}$ & BVS \\
\hline $\begin{array}{l}\text { Santos } \\
\mathrm{SM}^{4}\end{array}$ & $\begin{array}{l}201 \\
8\end{array}$ & $\begin{array}{l}\text { Two } \\
\text { Hemostasis } \\
\text { Methods After } \\
\text { Transradial } \\
\text { Cathetherizatio }\end{array}$ & SciELO \\
\hline
\end{tabular}




\begin{tabular}{|c|c|c|c|}
\hline & & $\begin{array}{l}\mathrm{n} \text { : THAMATIC- } \\
\text { protocol for a } \\
\text { randomized } \\
\text { clinical trial }\end{array}$ & \\
\hline $\begin{array}{l}\text { Jonatha } \\
\text { n S. } \\
\text { Roberts } \\
5\end{array}$ & $\begin{array}{l}201 \\
7\end{array}$ & $\begin{array}{l}\text { Comparison of } \\
\text { Hemostasis } \\
\text { Times With a } \\
\text { Kaolin-Based } \\
\text { Hemostatic Pad } \\
\text { (QuickKlot } \\
\text { Radial) vs } \\
\text { Mechanical } \\
\text { Compression } \\
\text { (TR Band) } \\
\text { Following } \\
\text { Transradial } \\
\text { Access: A Pilot } \\
\text { Prospective } \\
\text { Study }\end{array}$ & BVS \\
\hline $\begin{array}{l}\text { Barbosa } \\
\text { et al. }{ }^{6}\end{array}$ & $\begin{array}{l}201 \\
4\end{array}$ & $\begin{array}{l}\text { Utilização de } \\
\text { Pulseira } \\
\text { Compressora } \\
\text { Seletiva na } \\
\text { Prevenção da } \\
\text { Oclusão da } \\
\text { Artéria Radial } \\
\text { Após } \\
\text { Procedimento } \\
\text { Coronário } \\
\text { Invasivo }\end{array}$ & SciELO \\
\hline
\end{tabular}




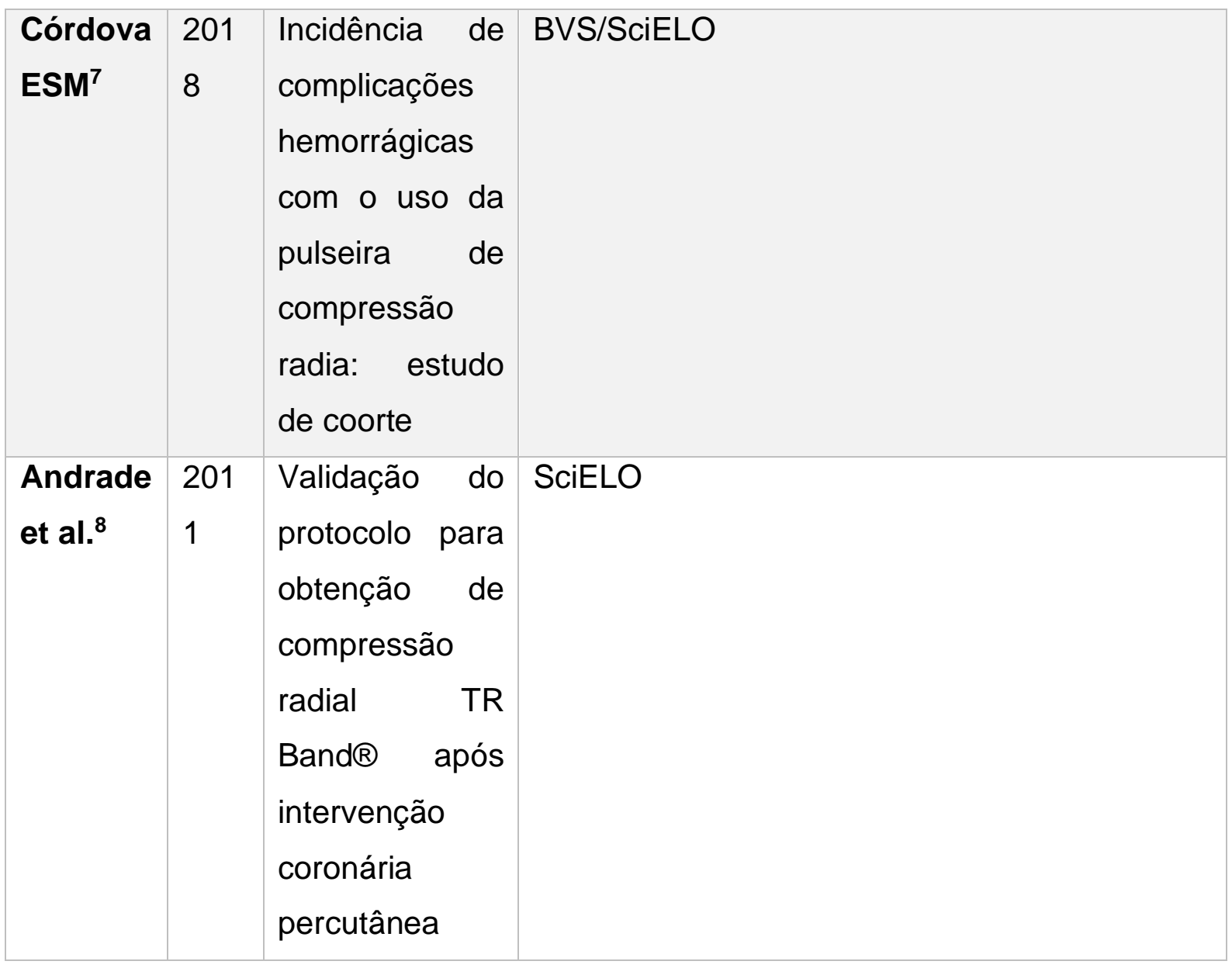

Fonte: autoria própria.2019

\section{DISCUSSÃO}

Os pacientes diagnosticados com Síndrome Coronariana Aguda submetidos a realização do cateterismo, ao receber a notícia do procedimento muitas vezes têm uma reação emocional de medo, ansiedade e insegurança, sendo estes fatores que podem interferir no processo. Assim torna-se importante destacar o quanto a tecnologia tem contribuído para a segurança e conforto do paciente. Este estudo revelou as vantagens do uso da pulseira compressora e a partir de 8 artigos examinados, evidenciou-se uma distribuição de artigos. Em relação às temáticas, os manuscritos abordavam principalmente as vantagens e comparações da pulseira compressora em relação a outro tipo de curativo e em relação à abordagem 
metodológica, observou-se a predominância de pesquisas qualitativas, do tipo descritivo, incluindo os estudos documentais. ${ }^{7}$

A pulseira de compressão radial TR Band® foi desenvolvida pela Terumo Medical, de fácil manuseio e higienização, e possibilita a sua reutilização, uma vantagem na redução de custos hospitalares, quando comparada a outras técnicas compressivas repercutindo em qualidade e segurança assistencial. ${ }^{7}$

Ainda que o valor das pulseiras de compressão varie de acordo com o fabricante e conforme acordo de venda com os diferentes hospitais, em nossa realidade estes dispositivos custam 20 vezes mais que o curativo manual com gaze ( $R \$ 50,00$ vs. $R \$$ $2,50)$. Porém, com o uso da pulseira, o paciente reduz a quantidade de dias que ficaria em uma unidade de internação, assim, diminuindo gastos maiores futuramente. ${ }^{3}$

De acordo com o presente estudo notou-se que a pulseira hemostática era mais usada em pacientes entre 57 anos de idade, sexo masculino e utilizava menos medicações adjuntas com a pulseira hemostática em relação ao curativo compressivo com gaze. ${ }^{3}$

Os pacientes permaneceram por 4 horas com a pulseira para compressão, tempo recomendado pelo fabricante. A partir da terceira hora, iniciou-se a desinflação do balão, mantendo-o conectado à seringa e controlando o êmbolo com o polegar, observando o sítio de punção. Pelo fato de o material da pulseira ser transparente, era possível a visualização a cada hora o local da punção. Uma vez que a hemostasia foi alcançada após a quarta hora, era retirada a pulseira, e os pacientes eram liberados para casa. $^{7}$

Uma das complicações vasculares locais pós ICP é a OAR, sendo esta particularmente prevalece e possivelmente ocorre até $10 \%$ dos procedimentos radiológicos. ${ }^{4}$

Através de testes realizados conclui-se que a pulseira reduz o risco de OAR, possibilitando reutilização da via. ${ }^{5}$ 
Santos SM afirma que em um ECR que comparou dois dispositivos diferentes para compressão da artéria radial, o dispositivo TR Band ${ }^{\mathrm{TM}}$ foi superior ao dispositivo Radistop ${ }^{\mathrm{TM}}$ em termos de conforto: $77 \%$ dos pacientes do grupo TR Band ${ }^{\mathrm{TM}}$ se sentiram confortáveis durante a compressão versus $61 \%$ daqueles alocados no Radistop ${ }^{\mathrm{TM}} .{ }^{4}$

Em escala crescente, vários hospitais estão implantando protocolos afim de padronizar o manejo do dispositivo e minimizar erros. ${ }^{8}$

Para garantir a assistência de enfermagem ao paciente que utiliza a TR Band® para obtenção da hemostasia com sucesso, deve-se seguir alguns cuidados específicos. O enfermeiro que atua no setor de hemodinâmica afim de prestar a assistência de enfermagem no procedimento de cateterismo, tem um protocolo a seguir, sendo ele estabelecido pelo próprio fabricante da TR Band®.

Diante desses cuidados estabelecidos, foi elaborado um BUNDLE, onde auxiliará os profissionais que prestam cuidados ao paciente em utilização da pulseira hemostática TR Band®.

Figura 1. Cuidados de enfermagem com a pulseira hemostática TR Band®.

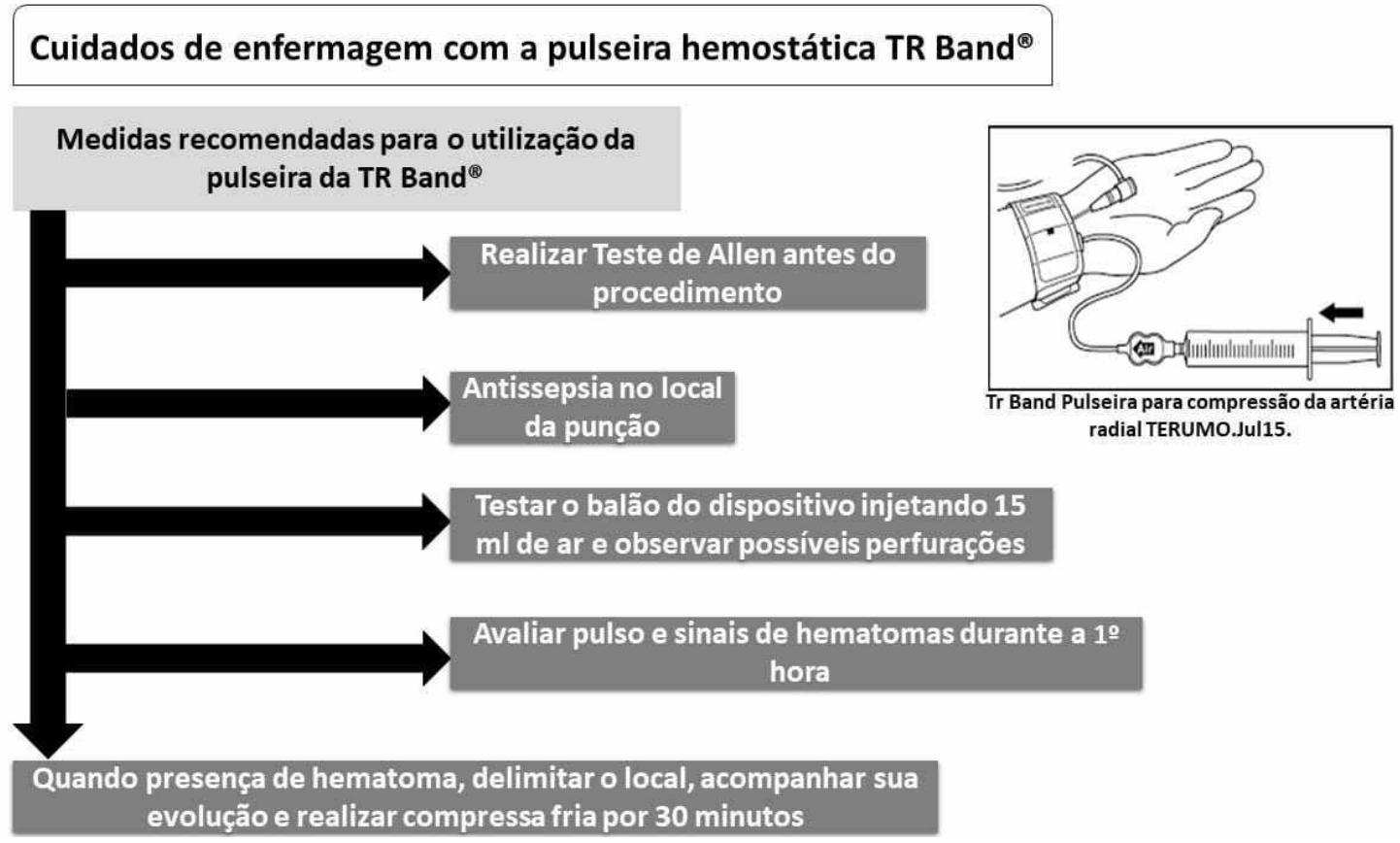

Fonte: autoria própria.2019 
Inicialmente, é preciso realizar o teste de Allen para avaliar a patência da artéria radial e em seguida é realizado a antissepsia e preparo do local para a punção. Em seguida, é testado o balão do dispositivo injetando ar e mergulhando em SF 0,9\% para observar possíveis perfurações. Feito isto, deve-se esvaziar o balão e secar o dispositivo, deixando-o preparado. Após o procedimento médico de ICP, será removido o introdutor arterial cerca de $2 \mathrm{~cm}$ e posicionado o dispositivo com a marcação no orifício da punção para melhor visualização de possíveis sangramentos. O balão deve ser insuflado com $15 \mathrm{ml}$ de ar com sua seringa própria, podendos ser acrescentado um volume adicional não ultrapassando de $18 \mathrm{ml}$ caso o volume inicial seja deficiente. $O$ processo de desinflação do balão inicia-se lentamente após 1 hora e 30 minutos do procedimento, sendo retirado de 3 a $5 \mathrm{ml}$ de ar a cada 30 minutos ou a cada 1 hora, variando de acordo com o paciente e/ou procedimento. Este processo deve ser gradual, pois se ocorrer um sangramento excessivo poderá existir uma dificuldade na visualização pela sujidade presente. Após o esvaziamento total do balão, deve-se confirmar se existe sinais de sangramento e ocluir o orifício com curativo. Salienta-se que a todo momento deve-se avaliar além dos sinais de sangramento, possíveis complicações como hematomas e pseudoaneurismas, caso apresente hematoma, deve-se delimitar o local, para avaliar se há crescimento de hematoma e realizar compressa fria por 30 minutos. $^{8}$

\section{CONCLUSÃO}

Durante a pesquisa, foram encontrados 13 artigos relacionados ao tema proposto e utilizados 8 artigos sobre as vantagens e desvantagens do uso da pulseira hemostática TR Band® e a assistência de enfermagem frente ao seu manejo. Por tratar-se de um dispositivo novo e recente no mercado e de uma fabricação importada, o número de artigos na língua portuguesa foi escasso referente a temática.

No que se refere à pulseira hemostática TR Band $\Theta$, alguns artigos foram encontrados, em suma na língua inglesa, um na língua russa e poucos na língua portuguesa.

Portanto, quanto aos dados de revisão coletados, concluímos que o uso da pulseira hemostática TR Band® é um dispositivo seguro e eficaz na hemostasia pós- 
cateterismo radial, levando em conta sua praticidade, tecnologia, conforto e segurança ao paciente.

\section{REFERÊNCIA}

1 Andrade MVA de et al. Validação de protocolo para obtenção de hemostasia com dispositivo de compressão radial TR Band ${ }^{\mathrm{TM}}$ após intervenção coronária percutânea. Rev. Bras. Cardiol. Invasiva [online]. 2011, vol.19, n.2, pp.184-188. ISSN 2179-8397. http://dx.doi.org/10.1590/S2179-83972011000200014.

2. Barbosa RA et al. Utilização de Pulseira Compressora Seletiva na Prevenção da Oclusão da Artéria Radial Após Procedimento Coronário Invasivo. Rev. Bras. Cardiol. Invasiva [online]. 2014, vol.22, n.2, pp.115-119. ISSN 21798397. http://dx.doi.org/10.1590/0104-1843000000020.

3. Cordova ESM et al. Incidência de complicações hemorrágicas com o uso de pulseira de compressão radial: estudo de coorte. Rev. esc. enferm. USP [online]. 2018, vol.52, e03410. Epub Dec 10, 2018. ISSN 0080-6234. http://dx.doi.org/10.1590/s1980220x2017041003410.

4. Dall'Orto CC et al. Angioplastia coronária nas indicações off-label : comparação das vias radial vs. femoral . Rev. Bras. Cardiol. Invasiva [online]. 2010, vol.18, n.2, pp.177184. ISSN 2179-8397. http://dx.doi.org/10.1590/S2179-83972010000200012.

5. Deulingh $\mathrm{JH}$ et al. A randomised controlled study of standard versus accelerated deflation of the Terumo radial band haemostasis decive after transradial diagnostic cardiac catheterisstion. European Journal of Cardiovascular Nursing , 2017, 16 (4), 344-351. https://doi.org/10.1177/1474515116672123.

6. Nóbrega ERA. Hemostasia da artéria radial pós cateterismo cardíaco: comparação randomizada do tempo de compressão e avaliação das complicações vasculares. DISSERTAÇÃO-Raquel-2016-Biblioteca-05-07-2016(1).pdf. https://repositorio.ufpe.br/handle/123456789/18942. 
7. Roberts JS; Nju J; Pastor-Cervantes JA. Comparison of Hemostasis Times With a Kaolin-Based Hemostatic Pad (QuikClot Radial) vs Mechanical Compression (TR Band) Following Transradial Access: A Pilot Prospective Study. J Invasive Cardiol, 2017, 29(10), 328-334.

8. Neto SA; Freitas JO; Berti SL; Jr JRC; Zbeid JAL. Comparação do curativo compressivo vs. Pulseira hemostática após cateterização por via radial. Rev. Bras. Cardiol. Invasiva [online]. 2015, vol 23, e $\quad 4$, pp 271-275. https://doi.org/10.1016/j.rbci.2017.01.001.

9. Santos SM; Rabelo-Silva ER; Aliti GB; Romero PS; Corrêa CL; Valle FH et al. Two HEmostasis Methods After Transradlal Catheterization: THEMATIC - protocol for a randomized clinical trial. Rev. Gaúcha Enferm. [Internet]. 2018 [cited 2019 Sep 19]; 39: e2017-0257. Available from:

http://www.scielo.br/scielo.php?script=sci_arttext\&pid=S198314472018000100433\&Ing=en. Epub Aug 02, 2018. http://dx.doi.org/10.1590/19831447.2018.2017-0257.

10. Pancholy SB, Patel TM. Effect of duration of hemostatic compression on radial artery occlusion after transradial access. Catheter Cardiovasc Interv. 2012;79:78-81.

11. Dai N; Xu DC; Hou L; Peng WH; Wei YD; Xu YW. (2014). A Comparison of 2 Devices for Radial Artery Hemostasis After Transradial Coronary Intervention. The Journal of cardiovascular nursing. 30. 10.1097/JCN.0000000000000115.

12. Ognerubov DV; Provatorov SI; Tereshchenko AS; Romasov IV; Pogorelova OA; Tripoten MI; Balakhonova TV; Merkulov EV; Samko AN. Rate of Complications at Early Removal of Compression Bandage After Transdarial Coronary Angiography. Cardiology. 2019; 59 (1): 79-83. https://doi.org/10.18087/cardio.2019.1.10218.

13. Rathore, S; Stables, RH; Pauriah, M et al. A Randomized Comparison of TR Band and Radistop Hemostatic Compression Decives After Transradial Coronary Intervention. Cateter Cardiovasc Interv 2010; 76: 660-7. [ PubMed] [ Google Acadêmico ] 
Enviado: Outubro, 2019.

Aprovado: Outubro, 2019. 